\title{
The structure of uniform discrete defective crystals
}

Received: date / Accepted: date

\begin{abstract}
In the continuum context, a uniform crystal has dislocation density tensor constant in space. A simple iteration procedure generates an infinite set of points which is associated with uniform defective crystals. When certain necessary conditions are satisfied, there is a minimum (non-zero) separation of points in this set, so the set is discrete. We describe the structure of such sets explicitly, and show in particular that any such set is either a simple lattice or a 4-lattice.
\end{abstract}

Keywords First keyword · Second keyword · More

\section{Introduction}

The motivation for this work is the following: in 1986 Davini [1] introduced a kinematical framework for a theory of defective crystals wherein three smooth linearly independent 'lattice vector fields' $\boldsymbol{\ell}_{1}, \boldsymbol{\ell}_{2}, \boldsymbol{\ell}_{3}$, were supposed to represent an 'averaged' atomic structure, with these three vector fields varying on a length scale coarser than atomic. The averaging procedure was not made precise. In order to set up the continuum mechanics appropriate to such a kinematical framework, one might reflect the discrete, atomistic, nature of the material (a 'crystalline material') by assuming that the continuum strain

P.C was partially supported by the Italian MIUR grant 'Modelli Matematici per la Scienza dei Materiali.

P. Cermelli

Dipartimento di Matematica, Università di Torino, Via C. Alberto 10, 10123 Torino, Italy

E-mail: paolo.cermelli@unito.it

G. P. Parry

School of Mathematical Sciences, University of Nottingham, Nottingham NG7 2RD, UK

E-mail: gareth.parry@nottingham.ac.uk . 
energy function has the symmetry of some underlying discrete structure. (Just as, in traditional linear elasticity theory, one imposes cubic or tetragonal symmetry, say, on the elastic moduli and thereby defines a particular linear elastic crystalline material. One does not enquire as to the scale of the associated underlying cubic or tetragonal lattice, when imposing such symmetries.)

There is a systematic way to associate a discrete structure with a set of smooth vector fields, even in the case where the vector fields represent defective crystalline materials, so that the vector fields do not commute, the Burgers vectors and dislocation density tensor are non zero, etc. This procedure was outlined in Parry [2]. When the material (regarded as a continuum) is defective, there is generally no elastic deformation of the material that 'straightens out' the lattice vector fields, even locally. Correspondingly, there is generally no (restriction of) such a deformation that maps the relevant discrete defective structure to a perfect lattice. However, it was shown in Parry [2] that when a uniform defective crystalline material has an underlying discrete structure, that structure is a 'multilattice'. Recall that a lattice (or 'simple lattice', or 'perfect lattice') $L$ is defined by

$$
L=\left\{\boldsymbol{x}: \boldsymbol{x}=n_{i} \boldsymbol{e}_{i}, n_{i} \in \mathbb{Z}, \quad i=1,2,3\right\},
$$

where $\boldsymbol{e}_{1}, \boldsymbol{e}_{2}, \boldsymbol{e}_{3}$ is a basis of $\mathbb{R}^{3}$. Then a multilattice $M$ is a superposition of a finite number of simple lattices, so there exist 'shift vectors' $\boldsymbol{p}_{1}, \boldsymbol{p}_{2} \ldots \boldsymbol{p}_{n}$ with $\boldsymbol{p}_{i}-\boldsymbol{p}_{j} \notin L$ if $i \neq j, i, j=1,2, \ldots n$, such that

$$
M=\left\{\boldsymbol{x}: \boldsymbol{x}=\boldsymbol{p}_{k}+L, L \text { is given by }(1.1), k=1,2, \ldots, n\right\} .
$$

In fact, a multilattice may have many representations of the form (1.2). Here we shall catalogue the various multilattices that arise as discrete structures corresponding to uniform defective crystals, and for each multilattice that arises, we give a representation (1.2) which is such that the integer $n$ is least (i.e. we give an 'essential' description of $M$, in the terminology of Pitteri \& Zanzotto [3]). Elsewhere, we shall investigate the geometrical symmetries of the multilattices with a view to prescribing material symmetries of corresponding continuum strain energy functions.

\section{Preliminaries}

Suppose, following Davini [1], that the geometric structure of a crystalline material in a region $\Omega \subseteq \mathbb{R}^{3}$ is specified by the prescription of three linearly independent smooth vector fields $\boldsymbol{\ell}_{a}, a=1,2,3$. Define the duals $\boldsymbol{d}^{a}, a=$ $1,2,3$ of these 'lattice vector fields' by

$$
\boldsymbol{d}^{a}(\boldsymbol{x}) \cdot \boldsymbol{\ell}_{b}(\boldsymbol{x})=\delta_{b}^{a}, \quad \boldsymbol{x} \in \Omega, \quad a, b,=1,2,3,
$$

where $\delta_{b}^{a}=1$ if $a=b, \delta_{b}^{a}=0$ otherwise. Also define the scalar field $n$ by

$$
n(\boldsymbol{x})=\boldsymbol{d}^{1}(\boldsymbol{x}) \cdot \boldsymbol{d}^{2}(\boldsymbol{x}) \wedge \boldsymbol{d}^{3}(\boldsymbol{x}), \quad \boldsymbol{x} \in \Omega .
$$


Then $n \neq 0$ in $\Omega$, by the linear independence of the lattice vector fields. The Burgers vectors of the theory of continuous distributions of dislocations are the three vector fields $\nabla \wedge \boldsymbol{d}^{a}, a=1,2,3$ (see Parry [2] for geometric interpretation), and the dislocation density tensor (ddt) $S^{a b}$ is defined by

$$
S^{a b}(\boldsymbol{x})=\frac{\nabla \wedge \boldsymbol{d}^{a}(\boldsymbol{x}) \cdot \boldsymbol{d}^{b}(\boldsymbol{x})}{n(\boldsymbol{x})}, \quad \boldsymbol{x} \in \Omega .
$$

Define the state $\Sigma$ of the material by

$$
\Sigma=\left\{\ell_{a}, \Omega ; a=1,2,3\right\},
$$

and let

$$
\Sigma^{*}=\left\{\ell_{a}^{*}, \Omega^{*} ; a=1,2,3\right\},
$$

be a different state of the same material. States $\Sigma, \Sigma^{*}$ are said to be elastically related to one another if there exists a diffeomorphism $\boldsymbol{u}: \Omega \rightarrow \Omega^{*}=\boldsymbol{u}(\Omega)$ such that

$$
\ell_{a}^{*}(\boldsymbol{u}(\boldsymbol{x}))=\nabla \boldsymbol{u}(\boldsymbol{x}) \boldsymbol{\ell}_{a}(\boldsymbol{x}), \quad \boldsymbol{x} \in \Omega
$$

One calculates from (8) that

$$
\left(\nabla \wedge \boldsymbol{d}^{a *}\right)(\boldsymbol{u}(\boldsymbol{x}))=[\operatorname{det} \nabla \boldsymbol{u}(\boldsymbol{x})]^{-1} \nabla \boldsymbol{u}(\boldsymbol{x}) \nabla \wedge \boldsymbol{d}^{a}(\boldsymbol{x}), \quad \boldsymbol{x} \in \Omega,
$$

where the function det represents the determinant. This gives that

$$
S^{a b *}(\boldsymbol{u}(\boldsymbol{x}))=S^{a b}(\boldsymbol{x}), \quad \boldsymbol{x} \in \Omega, \quad a, b=1,2,3 .
$$

Relation (10) is particularly important. It implies that the ddt is an elastic invariant, unchanged in elastic deformation (so that it is a measure of 'inelastic' change). It also follows that

$$
\underset{\Omega^{*}}{\operatorname{range}} S^{a b *}=\underset{\Omega}{\operatorname{range}} S^{a b} \text {. }
$$

Equation (11) provides a necessary condition that states $\Sigma, \Sigma^{*}$ be elastically related to one another (it is a more useful condition than (10), since the unknown deformation $\boldsymbol{u}$ does not appear).

One finds in Elzanowski and Parry [4], Parry [2], a proposal to adopt a particular constitutive form of strain energy function, for defective crystals, and that proposal motivates the need to consider states when the ddt is constant in $\Omega$. We shall refer to such states as uniform states. Then, by virtue of (10) (or (11)), if $\Sigma$ is a uniform state, and $\Sigma^{*}$ is elastically related to $\Sigma$, then $\Sigma^{*}$ is a uniform state. Moreover the ddt has the same value in $\Sigma$ and in $\Sigma^{*}$.

The crystalline states that we consider in this paper shall all be uniform states, and the discrete structures that we construct in later sections shall all 'derive from' uniform states.

Now from (5)

$$
\nabla \wedge \boldsymbol{d}^{a}=S^{a b} n \boldsymbol{\ell}_{b}
$$


so that in a uniform state, taking the divergence of each side of equation (12),

$$
0=S^{a b}\left(\varepsilon_{b r s} S^{r s}\right),
$$

where $\left(\varepsilon_{b r s}\right)$ is the permutation symbol. So there is a constraint on the value of the ddt, in a uniform state - either the ddt is symmetric, or the axial vector of the antisymmetric part is an eigenvector of the ddt. In fact equation (13) is an expression of the Jacobi identity for a Lie algebra with structure constants $\left(\varepsilon_{b r s} S^{a b}\right)$, see Parry [2] for details, and this observation leads us to investigate the group structure of uniform states, and the group properties of discrete structures associated with a given uniform state.

\section{Group structures of uniform states}

Suppose that the lattice vector fields $\ell_{a}, a=1,2,3$, in a state $\Sigma \equiv$ $\left\{\boldsymbol{\ell}_{a}, \mathbb{R}^{3} ; a=1,2,3\right\}$, have the following self-similarity property - there exists a diffeomorphism $\boldsymbol{\psi}: \mathbb{R}^{3} \rightarrow \mathbb{R}^{3}$ such that

$$
\ell_{a}(\boldsymbol{\psi}(\boldsymbol{x}))=\nabla \boldsymbol{\psi}(\boldsymbol{x}) \boldsymbol{\ell}(\boldsymbol{x})
$$

(The point is that the fields on the left hand side of (14) are the same fields as on the right hand side of (14), by contrast with (8)). Then from (10)

$$
S^{a b}(\boldsymbol{\psi}(\boldsymbol{x}))=S^{a b}(\boldsymbol{x}) .
$$

If, further, given arbitrary points $\boldsymbol{x}_{0}, \boldsymbol{\psi}_{0} \in \mathbb{R}^{3}$, one can find $\boldsymbol{\psi}$ such that $\boldsymbol{\psi}\left(\boldsymbol{x}_{0}\right)=\boldsymbol{\psi}_{0}$ and (14) holds, then it follows from (15) that state $\Sigma$ is uniform.

In fact, according to Pontryagin [5], Theorem 85, the necessary and sufficient condition that (14) has a solution $\boldsymbol{\psi}$ satisfying $\boldsymbol{\psi}\left(\boldsymbol{x}_{0}\right)=\boldsymbol{\psi}_{0}$, with $\boldsymbol{x}_{0}, \boldsymbol{\psi}_{0}$ arbitrary, in some domain, is that the ddt is uniform there. Therefore (14) holds for an given uniform state, and one may assume that, if $\boldsymbol{x}_{0}, \boldsymbol{\psi}_{0}$ are given, then $\boldsymbol{\psi}\left(\boldsymbol{x}_{0}\right)=\boldsymbol{\psi}_{0}$.

This strong statement can be interpreted as the analogue, for the case of defective crystals, of the 'translational self-similarity' of a perfect lattice. Thus, if the state $\Sigma=\left\{\boldsymbol{e}_{a}, \mathbb{R}^{3} ; a=1,2,3\right\}$ is given, the translation $\boldsymbol{\psi} \equiv$ $\left(\boldsymbol{x}-\boldsymbol{x}_{0}\right)+\boldsymbol{\psi}_{0}$ is the required solution of (14).

Even more is true. Suppose that $\Sigma$ is uniform, that

$$
\ell_{a}(\mathbf{0})=e_{a}
$$

and consider the relation

$$
\ell_{a}(\boldsymbol{\psi}(\boldsymbol{x}, \boldsymbol{p}))=\nabla_{1} \boldsymbol{\psi}(\boldsymbol{x}, \boldsymbol{p}) \boldsymbol{\ell}_{a}(\boldsymbol{x})
$$

$\boldsymbol{p} \in \mathbb{R}^{3}$, when $\nabla_{1} \boldsymbol{\psi}$ denotes the gradient with respect to the first argument of the function $\psi$. According to Pontryagin [5] one may find a unique solution of (17) which is invertible in both arguments and has the properties

$$
\boldsymbol{\psi}(\mathbf{0}, \boldsymbol{p})=\boldsymbol{\psi}(\boldsymbol{p}, \mathbf{0})=\boldsymbol{p}, \quad \boldsymbol{\psi}(\boldsymbol{x}, \boldsymbol{\psi}(\boldsymbol{y}, \boldsymbol{z}))=\boldsymbol{\psi}(\boldsymbol{\psi}(\boldsymbol{x}, \boldsymbol{y}), \boldsymbol{z})
$$


So the solution of (17) defines a Lie group composition function - it provides an associative 'multiplication' of points in the domain of the lattice vector fields, and the origin acts as the group identity element. Note that, for the perfect 'continuous lattice' $\Sigma=\left\{\boldsymbol{e}_{a}, \mathbb{R}^{3} ; a=1,2,3\right\}, \boldsymbol{\psi} \equiv \boldsymbol{x}+\boldsymbol{p}$ is the solution of (17) that also satisfies (18).

Conversely, suppose that a function $\psi: \mathbb{R}^{3} \times \mathbb{R}^{3} \rightarrow \mathbb{R}^{3}$ that satisfies (18) is given. Then one may check that the vector fields $\boldsymbol{\ell}_{a}, a=1,2,3$, defined by

$$
\boldsymbol{\ell}_{a}(\boldsymbol{x})=\nabla_{1} \boldsymbol{\psi}(\mathbf{0}, \boldsymbol{x}) \boldsymbol{e}_{a},
$$

satisfy both (16) and (17). The dual vector fields corresponding to (19) are

$$
\boldsymbol{d}^{a}(\boldsymbol{x})=\nabla_{1} \psi^{a}\left(\boldsymbol{x}, \boldsymbol{x}^{-1}\right),
$$

where $\boldsymbol{\psi}=\left(\psi^{a}\right)$, and $\boldsymbol{\psi}\left(\boldsymbol{x}, \boldsymbol{x}^{-1}\right)=\mathbf{0}$ defines $\boldsymbol{x}^{-1}$.

In the Lie group literature, when (17) holds, the fields $\boldsymbol{\ell}_{a}, a=1,2,3$, are said to be right invariant (with respect to $\boldsymbol{\psi}$ ). Clearly, if $\boldsymbol{\ell}_{a}, a=1,2,3$, is some set of right invariant fields, then $\gamma_{a}^{b} \ell_{a}$ (where $\gamma=\left(\gamma_{a}^{b}\right)$ is a non singular $3 \times 3$ real matrix) is also a set of right invariant fields (with respect to the same $\boldsymbol{\psi}$, of course). Let $\boldsymbol{\nu}$ be an arbitrary right invariant field, so that

$$
\boldsymbol{\nu}(\boldsymbol{\psi}(\boldsymbol{x} \cdot \boldsymbol{p}))=\nabla_{1} \boldsymbol{\psi}(\boldsymbol{x}, \boldsymbol{p}) \boldsymbol{\nu}(\boldsymbol{x}) .
$$

Then, using (18) and (21), and writing $\boldsymbol{\nu}(0)=\nu^{a} \boldsymbol{e}_{a}$,

$$
\begin{aligned}
\boldsymbol{\nu}(\boldsymbol{x}) & =\boldsymbol{\nu}(\boldsymbol{\psi}(\mathbf{0}, \boldsymbol{x}))=\nabla_{1} \boldsymbol{\psi}(\mathbf{0}, \boldsymbol{x}) \boldsymbol{\nu}(\mathbf{0}) \\
& =\nu^{a}\left(\nabla_{1} \boldsymbol{\psi}(\mathbf{0}, \boldsymbol{x}) \boldsymbol{e}_{a}\right) \\
& =\nu^{a} \boldsymbol{\ell}_{a}(\boldsymbol{x}),
\end{aligned}
$$

if we define $\boldsymbol{\ell}_{a}(\boldsymbol{x})$ through (19). Therefore the particular right invariant fields defined by (19) provide a basis for the vector space of right invariant fields (with respect to $\psi$ ).

Finally, let $\Sigma$ be a uniform state and suppose that

$$
\boldsymbol{\ell}_{a}(\mathbf{0})=\boldsymbol{\ell}_{a}, \quad a=1,2,3 .
$$

Then it is clear that (17) has a solution $\boldsymbol{\psi}$ which satisfies (18). So let $\Gamma \equiv\left(\gamma_{a}^{b}\right)$ be the real $3 \times 3$ matrix with rows $\ell_{1}, \ell_{2}, \ell_{3}$, and define fields $\ell_{a}^{\prime}$ by

$$
\gamma_{a}^{b} \ell_{b}^{\prime}(\boldsymbol{x})=\boldsymbol{\ell}_{a}(\boldsymbol{x}), \quad \boldsymbol{x} \in \Omega .
$$

Then $\gamma_{a}^{b} \ell_{b}^{\prime}(\mathbf{0})=\boldsymbol{\ell}_{a}$, which implies that $\boldsymbol{\ell}_{b}^{\prime}(\mathbf{0})=\boldsymbol{e}_{b}$. So the fields $\boldsymbol{\ell}_{a}^{\prime}$ satisfy (16) and (17) for some unique composition function $\boldsymbol{\psi}$. It follows that (17) holds for the fields $\ell_{a}$ and composition function $\boldsymbol{\psi}$ so found. Also, it is clear that one may express fields that satisfy both (23) and (17) in terms of the basis right invariant fields:

$$
\boldsymbol{\ell}_{a}(\boldsymbol{x})=\nabla_{1} \boldsymbol{\psi}(\mathbf{0}, \boldsymbol{x}) \boldsymbol{\ell}_{a}(\mathbf{0}) \equiv \nabla_{1} \boldsymbol{\psi}(\mathbf{0}, \boldsymbol{x}) \boldsymbol{\ell}_{a} .
$$

Note that if $S$ is the ddt of the basis right invariant fields, which satisfy (16) and (17), if $\Gamma$ is defined as above, and if we denote by $\bar{S}$ the ddt of fields $\ell_{a}^{\prime}$ that satisfy (23) and (24), then

$$
\bar{S}=(\operatorname{det} \Gamma)^{-1} \Gamma^{T} S \Gamma .
$$




\section{The underlying discrete structure}

Given a uniform state $\Sigma$, we generate a set of points $S_{\Sigma}$ by the following iterative procedure. Choose $\boldsymbol{x}=\mathbf{0}$ as the initial point, construct six points denoted $e^{\ell_{a}}, e^{-\ell_{a}}, a=1,2,3$, by flow through times $+1,-1$ along the three lattice vector fields. Continue in this way (cf. Figure 2).

To be precise, suppose that (16) holds and let $\boldsymbol{\psi}$ be the solution of (17), so that (18) also holds.

Let $\boldsymbol{x}_{0}$ be arbitrary and consider the flow $\boldsymbol{x}: \mathbb{R} \rightarrow \mathbb{R}^{3}$ defined by the solution of the ordinary differential equation

$$
\frac{d \boldsymbol{x}}{d t}=\boldsymbol{\ell}_{a}(\boldsymbol{x}(t)), \quad \boldsymbol{x}(0)=\boldsymbol{x}_{0}
$$

Let $\exp \left(t \ell_{a}\right): \mathbb{R}^{3} \rightarrow \mathbb{R}^{3}$ be the mapping defined by

$$
\exp \left(t \ell_{a}\right) \boldsymbol{x}(0)=\boldsymbol{x}(t) .
$$

Let $e^{t \ell_{a}} \in \mathbb{R}^{3}$ be the points defined by

$$
e^{t \ell_{a}}=\exp \left(t \ell_{a}\right) \mathbf{0}
$$

Then the three points obtained by flow through time +1 along the three lattice vector fields are $e^{\ell_{a}}$, according to (29). Olver [6], for example, shows that

$$
\exp \left(t \boldsymbol{\ell}_{a}\right) \exp \left(s \boldsymbol{\ell}_{a}\right)=\exp \left[(s+t) \boldsymbol{\ell}_{a}\right],\left[\exp \left(t \boldsymbol{\ell}_{a}\right)\right]^{-1}=\exp \left(-t \boldsymbol{\ell}_{a}\right) .
$$

The point $\boldsymbol{p}$ obtained by flow through time -1 from $\boldsymbol{x}_{0}$ as initial point is (by definition) such that

$$
\frac{d \boldsymbol{x}}{d t}=\boldsymbol{\ell}_{a}(\boldsymbol{x}(t)), \quad \boldsymbol{x}(-1)=\boldsymbol{p}, \quad \boldsymbol{x}(0)=\boldsymbol{x}_{0} .
$$

So from $(28)$

$$
\boldsymbol{p}=\exp \left(-\boldsymbol{\ell}_{a}\right) \boldsymbol{x}_{0}
$$

and hence the flow through time -1 , starting at $\mathbf{0}$, yields the three points $e^{-\ell_{a}}$ (from (29)).

Now it is a standard result, reproduced by Parry [2], that

$$
\exp \left(t \ell_{a}\right) \boldsymbol{x}=\boldsymbol{\psi}\left(e^{t \ell_{a}}, \boldsymbol{x}\right) .
$$

That is, flow for time $t$ along the vector field $\boldsymbol{\ell}_{a}$, with initial point $\boldsymbol{x}$, produces the point obtained by group multiplication of points $e^{t \ell_{a}}, \boldsymbol{x}$. Also, using (29), (30), (33), we have

$$
\mathbf{0}=\exp \left(-t \boldsymbol{\ell}_{a}\right) \exp \left(t \boldsymbol{\ell}_{a}\right) \mathbf{0}=\exp \left(-t \boldsymbol{\ell}_{a}\right) e^{t \boldsymbol{\ell}_{a}}=\boldsymbol{\psi}\left(e^{-t \boldsymbol{\ell}_{a}}, e^{t \boldsymbol{\ell}_{a}}\right),
$$

so that $e^{-t \ell_{a}}$ is the group inverse of $e^{t \ell_{a}}$, and in particular

$$
e^{-\ell_{a}}=\left(e^{\ell_{a}}\right)^{-1} .
$$


So the first step of iteration procedure produces the six points $e^{\ell_{a}},\left(e^{\ell_{a}}\right)^{-1}$, $a=1,2,3$. By virtue of (33), the next step produces the thirty six multiples of these six group elements, etc. Moreover the origin acts as group identity element, from (18).Let $G$ be the free group generated by the three elements $e^{\ell_{a}}, a=1,2,3$, so $G$ is a subgroup of the Lie group $H$ that corresponds to the action of the composition function $\boldsymbol{\psi}$ on $\mathbb{R}^{3}$. Also $S_{\Sigma}$ is the orbit of $\mathbf{0} \in \mathbb{R}^{3}$ under the multiplicative action of $G$. The issue of whether or not $S_{\Sigma}$ is a discrete set, that is whether or not there is a nonzero minimum distance between the points of $S_{\Sigma}$, turns out to depend just on the value of the ddt. Following Thurston [7], assuming that the points $e^{\ell_{a}}, a=1,2,3$, are 'close enough' to the origin (see Parry [2] for details), if $S_{\Sigma}$ is discrete then the ddt must have the form.

$$
S^{a b}=\lambda \nu^{a} \nu^{b}, \quad a, b=1,2,3,
$$

$\boldsymbol{\nu}=\left(\nu^{a}\right) \in \mathbb{R}^{3}, \lambda \in \mathbb{R}$. In that case the corresponding Lie group $H$ is called nilpotent, in the literature.

We shall confine attention in this paper to the case where (36) holds, and show that if $S_{\Sigma}$ is discrete, then $\lambda, \nu^{a}, a=1,2,3$, may be assumed to be rational. We then show that these necessary conditions that $S_{\Sigma}$ be discrete are also sufficient for that purpose by giving the explicit multilattice structure of $S_{\Sigma}$ for each choice of (rational) $\lambda, \nu^{a}$ in (36). Note that $\left(S^{a b}\right)$ is symmetric, when (36) holds, so that (13) also holds.

\section{Behaviour under elastic deformation}

The ddt is an elastic invariant. It is also true that the iteration process outlined above 'commutes' with elastic deformation. That is, if (uniform) state $\Sigma$ is elastically related to state $\Sigma^{*}$ via (8), then

$$
S_{\Sigma^{*}}=\boldsymbol{u}\left(S_{\Sigma}\right) .
$$

The flexibility afforded by (37) will allow us to show, later, that $S_{\Sigma}$ is a multilattice in certain 'canonical states', when (36) holds. In this section we first of all prove (37), then consider the effect of elastic deformation on various quantities introduced in sections 3 and 4 .

First, let $\Sigma, \Sigma^{*}$ be elastically related states, so (8) holds for some diffeomorphism $\boldsymbol{u}$. It is sufficient, to prove that (37) holds, to show that if

$$
\dot{\boldsymbol{x}}=\boldsymbol{\ell}_{a}(\boldsymbol{x}), \boldsymbol{x}(0)=\boldsymbol{x}_{0}, \boldsymbol{x}(t)=\boldsymbol{x}_{t}
$$

and if

$$
\dot{\boldsymbol{y}}=\boldsymbol{\ell}_{a}^{*}(\boldsymbol{y}), \boldsymbol{y}(0)=\boldsymbol{u}\left(\boldsymbol{x}_{0}\right), \boldsymbol{y}(t)=\boldsymbol{y}_{t},
$$

then

$$
\boldsymbol{y}_{t}=\boldsymbol{u}\left(\boldsymbol{x}_{t}\right)
$$

But (39) has unique solution

$$
\boldsymbol{y}(t)=\boldsymbol{u}(\boldsymbol{x}(t)),
$$


because

$$
\dot{\boldsymbol{y}}=\nabla \boldsymbol{u}(\boldsymbol{x}) \dot{\boldsymbol{x}}=\nabla \boldsymbol{u}(\boldsymbol{x}) \boldsymbol{\ell}_{a}(\boldsymbol{x})=\boldsymbol{\ell}_{a}^{*}(\boldsymbol{u}(\boldsymbol{x}))=\boldsymbol{\ell}_{a}^{*}(\boldsymbol{y}),
$$

via (8) and (38). So (40) is proven, and (37) holds.

We record, from Parry [2], that if (8) and (17) hold, so

$$
\boldsymbol{\ell}_{a}^{*}(\boldsymbol{u}(\boldsymbol{x}))=\nabla \boldsymbol{u}(\boldsymbol{x}) \boldsymbol{\ell}_{a}(\boldsymbol{x}), \boldsymbol{\ell}_{a}(\boldsymbol{\psi}(\boldsymbol{x}, \boldsymbol{p}))=\nabla_{1} \boldsymbol{\psi}(\boldsymbol{x}, \boldsymbol{p}) \boldsymbol{\ell}_{a}(\boldsymbol{x}),
$$

(with no restriction on the values of $\boldsymbol{\ell}_{a}(\mathbf{0})$ ), then

$$
\boldsymbol{\ell}_{a}^{*}\left(\boldsymbol{\psi}^{*}\left(\boldsymbol{x}^{*}, \boldsymbol{p}^{*}\right)\right)=\nabla_{1} \boldsymbol{\psi}^{*}\left(\boldsymbol{x}^{*}, \boldsymbol{p}^{*}\right) \boldsymbol{\ell}_{a}^{*}\left(\boldsymbol{x}^{*}\right),
$$

where

$$
\boldsymbol{\psi}^{*}\left(\boldsymbol{x}^{*}, \boldsymbol{y}^{*}\right) \equiv \boldsymbol{u}\left(\boldsymbol{\psi}\left(\boldsymbol{u}^{-1}\left(\boldsymbol{x}^{*}\right), \boldsymbol{u}^{-1}\left(\boldsymbol{y}^{*}\right)\right) .\right.
$$

Moreover, if $\boldsymbol{u}(\mathbf{0})=\mathbf{0}$, then (18) implies that

$$
\boldsymbol{\psi}^{*}\left(\mathbf{0}, \boldsymbol{p}^{*}\right)=\boldsymbol{\psi}^{*}\left(\boldsymbol{p}^{*}, \mathbf{0}\right)=\boldsymbol{p}^{*}, \boldsymbol{\psi}^{*}\left(\boldsymbol{x}^{*}, \boldsymbol{\psi}^{*}\left(\boldsymbol{y}^{*}, \boldsymbol{z}^{*}\right)\right)=\boldsymbol{\psi}^{*}\left(\boldsymbol{\psi}^{*}\left(\boldsymbol{x}^{*}, \boldsymbol{y}^{*}\right), \boldsymbol{z}^{*}\right) .
$$

Also, the right invariant field, $\boldsymbol{\nu}^{*}$, satisfying (21) with composition function $\boldsymbol{\psi}^{*}$, is the elastic image of the right invariant field $\boldsymbol{v}$, corresponding to $\boldsymbol{\psi}$, satisfying (21). The corresponding exponential mappings $\exp \left(t \boldsymbol{v}^{*}\right)$, and points $e^{t \boldsymbol{v}^{*}}$, satisfy

$$
\exp \left(t \boldsymbol{v}^{*}\right) \boldsymbol{x}^{*}=\boldsymbol{u}(\exp (t \boldsymbol{v}) \boldsymbol{x}), \quad e^{t \boldsymbol{v}^{*}}=\boldsymbol{u}\left(e^{t \boldsymbol{v}}\right)
$$

if $\boldsymbol{x}^{*}=\boldsymbol{u}(\boldsymbol{x})$.

Let us consider the effect of the diffeomorphism $\boldsymbol{u}$ on the basis right invariant fields

$$
\boldsymbol{\ell}_{a}(\boldsymbol{x})=\nabla_{1} \boldsymbol{\psi}(\mathbf{0}, \boldsymbol{x}) \boldsymbol{e}_{a} .
$$

Define basic right invariant fields with respect to composition function $\boldsymbol{\psi}^{*}$ by

$$
\boldsymbol{\ell}_{a}^{+}(\boldsymbol{x})=\nabla_{1} \boldsymbol{\psi}^{*}(\mathbf{0}, \boldsymbol{x}) \boldsymbol{e}_{a} .
$$

According to Parry [2], the elastic image of a right invariant field is right invariant, so there exist constants $p_{a}^{\cdot b}, a, b=1,2,3$ such that

$$
\boldsymbol{\ell}_{a}^{*}(\boldsymbol{u}(\boldsymbol{x})) \equiv \nabla \boldsymbol{u}(\boldsymbol{x}) \boldsymbol{\ell}_{a}(\boldsymbol{x})=p_{a}^{\cdot b} \boldsymbol{\ell}_{a}^{+}(\boldsymbol{u}(\boldsymbol{x}))
$$

Evaluating at $\mathbf{0}$,

$$
\nabla \boldsymbol{u}(\mathbf{0}) \boldsymbol{e}_{a}=p_{a}^{\cdot b} \boldsymbol{e}_{b}
$$

so the matrix $P \equiv\left(p_{a}^{\cdot b}\right)$ is given by

$$
P=(\nabla \boldsymbol{u}(\mathbf{0}))^{T},
$$

where $T$ denotes the transpose. Let $S, S^{+}$be the ddt for states with lattice vector fields $\boldsymbol{\ell}_{a}, \boldsymbol{\ell}_{a}^{+}, a=1,2,3$ respectively. Then, from $(50)_{1}$, the ddt for the state corresponding to lattice vector fields $\ell_{a}^{*}, a=1,2,3$ is $S$, and by $(50)_{2}$ and an analogue of (26),

$$
S=(\operatorname{det} P) P^{-T} S^{+} P^{-1},
$$

hence

$$
S^{+}=(\operatorname{det} P)^{-1} P^{T} S P .
$$




\section{Canonical states}

Given the value of the ddt $S$, and a corresponding uniform state, then by elastic deformation of that state one may construct another (uniform) state $\Sigma$ where the lattice vector fields have a certain canonical form (Pontryagin [5]). In such a canonical state, the set of points determined by flow along any right invariant field is a straight line in $\mathbb{R}^{3}$ - in particular flow along any basis right invariant field produces a straight line.

The necessary and sufficient condition that right invariant lattice vector fields $\ell_{a}, a=1,2,3$ have this defining property of canonical states is that

$$
\ell_{a}^{k}(\boldsymbol{x}) x^{a}=x^{k} \quad a, k=1,2,3, \quad \boldsymbol{x} \in \mathbb{R}^{3},
$$

(or equivalently $d_{j}^{a}(\boldsymbol{x}) x^{j}=x^{a}$ ). Notice that by differentiating (55) with respect to $\boldsymbol{x}$, and then setting $\boldsymbol{x}=\mathbf{0}$, one may deduce that $\boldsymbol{\ell}_{a}(0)=\boldsymbol{e}_{a}$, so that right invariant fields that satisfy (55) are basis right invariant fields. Equation (55) may be solved for the fields $\boldsymbol{\ell}_{a}, a=1,2,3$, given $S$, in the following manner: let

$$
w_{j}^{i}(t) \equiv w_{j}^{i}(t, \boldsymbol{x}) \equiv t d_{j}^{i}(t \boldsymbol{x}), \quad t \in \mathbb{R}, \boldsymbol{x} \in \mathbb{R}^{3} .
$$

Pontryagin [5] shows that when (55) holds for basis right invariant field $\boldsymbol{\ell}_{a}$, then

$$
\frac{d w_{j}^{i}}{d t}=\delta_{j}^{i}+c_{\alpha \beta}^{i} x^{\alpha} w_{j}^{\beta}, \quad w_{j}^{i}(0)=0
$$

where $c_{\alpha \beta}^{i} \equiv \varepsilon_{\alpha \beta \gamma} S^{i \gamma}$. Conversely when (57) holds and one defines $d_{j}^{i}(x) \equiv$ $w_{j}^{i}(1, x) \equiv w_{j}^{i}(1)$ (cf. (56)), then (55) holds - that is, the basis right invariant fields are constructed by this procedure. One may find the corresponding composition function by solving (17), given the lattice vector fields.

Now let us recognise that the functions $w_{j}^{i}$ in (57) also depend on $S$, and write

$$
w_{j}^{i}(t) \equiv w_{j}^{i}(t, \boldsymbol{x}, S) .
$$

Let $\ell_{a}$ be basis right invariant fields corresponding to composition function $\boldsymbol{\psi}$, ddt $S$. Define

$$
\tilde{\boldsymbol{\ell}}_{a}(\boldsymbol{x})=\left(\gamma^{-T}\right)_{a}^{b} \boldsymbol{\ell}_{b}(\boldsymbol{x}), \quad \boldsymbol{x} \in \mathbb{R}^{3}
$$

where $\gamma$ is the gradient of a homogeneous elastic deformation that maps fields $\tilde{\ell}_{a}$ (composition function $\boldsymbol{\psi}$, ddt $\widetilde{S}$ ) to fields $\boldsymbol{\ell}_{a}^{*}$ (composition function defined by $(45)$, ddt $S)$, so

$$
\boldsymbol{\ell}_{a}^{*}(\gamma \boldsymbol{x})=\gamma \tilde{\boldsymbol{\ell}}_{a}(x)=\gamma\left[\left(\gamma^{-T}\right)_{a}^{b} \boldsymbol{\ell}_{b}(\boldsymbol{x})\right]
$$

By construction

$$
\boldsymbol{\ell}_{a}^{*}(0)=\gamma\left[\left(\gamma^{-T}\right)_{a}^{b} \boldsymbol{e}_{b}\right]=\boldsymbol{e}_{a}, \quad a=1,2,3
$$

Notice that by $(60)_{2},(55)$,

$$
\begin{aligned}
\ell_{a}^{* k}(\gamma \boldsymbol{x})(\gamma \boldsymbol{x})^{a} & =\gamma_{p}^{k}\left[\left(\gamma^{-T}\right)_{a}^{b} \ell_{b}^{p}(\boldsymbol{x})\right] \gamma_{j}^{a} x^{j} \\
& =\gamma_{p}^{k} \ell_{j}^{p}(\boldsymbol{x}) x^{j}=(\gamma \boldsymbol{x})^{k}
\end{aligned}
$$


so that, either from $(61)_{2}$ and the properties of right invariant fields, or from (62) and (55), the fields $\ell_{a}^{*}$ are basis right invariant fields corresponding to ddt $(\operatorname{det} \gamma)^{-1} \gamma S \gamma^{T}$, from (26). It follows that (60) may be written, in a notation analogous to that introduced in (58), as

$$
\boldsymbol{\ell}_{a}^{i}\left(\gamma \boldsymbol{x},(\operatorname{det} \gamma)^{-1} \gamma S \gamma^{T}\right)=\gamma_{j}^{i} \ell_{b}^{j}(\boldsymbol{x}, S)\left(\gamma^{-T}\right)_{a}^{b}
$$

where $\boldsymbol{\ell}_{b}(\boldsymbol{x}, S)$ are basis right invariant fields corresponding to ddt $S$. In fact one may verify directly from (57) that

$$
w_{j}^{i}\left(t, \gamma \boldsymbol{x},(\operatorname{det} \gamma)^{-1} \gamma S \gamma^{T}\right)=\gamma_{b}^{a} w_{k}^{b}(t, \boldsymbol{x}, S)\left(\gamma^{-1}\right)_{i}^{k}, \quad t \in \mathbb{R},
$$

and deduce from this equation that (63) holds.

\section{Discrete structures}

Our task is to study discrete structures generated according to the iteration procedure outlined in section 4 , with $S$ nilpotent. Since iteration commutes with elastic deformation, it is sufficient to study canonical states which have nilpotent ddt. So we may assume that (36), (55), (56), (57) hold. One verifies that the solution of (57), when (36) holds, gives

$$
\boldsymbol{\ell}_{a}(\boldsymbol{x})=\boldsymbol{e}_{a}+\frac{1}{2} \lambda \boldsymbol{v}\left(\boldsymbol{x} \wedge \boldsymbol{v} \cdot \boldsymbol{e}_{a}\right), \quad \boldsymbol{d}^{a}=\boldsymbol{e}^{a}+\frac{1}{2} \lambda v^{a}(\boldsymbol{v} \wedge \boldsymbol{x}),
$$

and that the corresponding composition function is

$$
\boldsymbol{\psi}(\boldsymbol{x}, \boldsymbol{y})=\boldsymbol{x}+\boldsymbol{y}+\frac{1}{2}(\boldsymbol{v} \cdot \boldsymbol{x} \wedge \boldsymbol{y}) \boldsymbol{\sigma}, \quad \boldsymbol{x}, \boldsymbol{y} \in \mathbb{R}^{3},
$$

where we define

$$
\boldsymbol{\sigma} \equiv \lambda \boldsymbol{v}
$$

Notice that given $S$, the choice of $\lambda, \boldsymbol{v}$ such that $S=\lambda \boldsymbol{v} \otimes \boldsymbol{v}$ is not unique.

Proposition 1 If $\Sigma \equiv\left\{\boldsymbol{\ell}_{a}, \Omega ; a=1,2,3\right\}$, with $\boldsymbol{\ell}_{a}$ given by (65), and if $S_{\Sigma}$ is discrete, then one may choose $\boldsymbol{v} \in \mathbb{R}^{3}$ such that its components are relatively prime integers, and choose $\lambda$ to be rational. With this choice of $\lambda, \boldsymbol{v}$, the vectors $\lambda \boldsymbol{v}, 2 \boldsymbol{e}_{1}, 2 \boldsymbol{e}_{2}, 2 \boldsymbol{e}_{3}$ are translations of $S_{\Sigma}$.

Proof According to Parry [2], if $\boldsymbol{x} \in S_{\Sigma}$, then $\boldsymbol{x}+\lambda \boldsymbol{v}\left(n_{c} v^{c}\right) \in S_{\Sigma}$ where $v^{1}, v^{2}, v^{3}$ are the components of $\boldsymbol{v}$, and $n_{1}, n_{2}, n_{3}$ are arbitrary integers. Suppose for the sake of contradiction that the ratio of some two components of $\boldsymbol{v}$ is irrational, say $v^{2} / v^{1} \notin \mathbb{Q}$. Then, from the result quoted, since $\mathbf{0} \in S_{\Sigma}$, $\lambda \boldsymbol{v}\left(n_{1} v^{1}+n_{2} v^{2}\right)=\lambda \boldsymbol{v} v^{1}\left(n^{1}-n^{2} \theta\right) \in S_{\Sigma}$ where $\theta \equiv-v^{2} / v^{1} \notin \mathbb{Q}$. But one may find a sequence of pairs of integers $n_{m}^{1}, n_{m}^{2}$ such that $\left|n_{m}^{1}-n_{m}^{2} \theta\right| \rightarrow 0$ as $m \rightarrow \infty$ (according to Rose [8] p123, there are infinitely many convergents $n_{m}^{1} / n_{m}^{2}$ to irrational $\theta$ which satisfy $\left|\frac{n_{m}^{1}}{n_{m}^{2}}-\theta\right|<\frac{5^{-1 / 2}}{\left(n_{m}^{2}\right)^{2}}$, with $n_{m}^{2} \rightarrow \infty$ as $m \rightarrow \infty)$. It follows that $\mathbf{0}$ is an accumulation point of $S_{\Sigma}$, which is a contradiction. So $\boldsymbol{v}$ may be written in the form $\boldsymbol{v}=\kappa\left(v^{1^{\prime}}, v^{2^{\prime}}, v^{3^{\prime}}\right)$, where each of $v^{1^{\prime}}, v^{2^{\prime}}, v^{3^{\prime}}$ is rational. Clearly, one may assume further that $v^{1^{\prime}}, v^{2^{\prime}}, v^{3^{\prime}}$ 
are relatively prime integers. Next, it is an easy extension of Euclid's algorithm to show that there exist integers $n_{1}^{\prime}, n_{2}^{\prime}, n_{3}^{\prime}$ such that $n_{c}^{\prime} v^{c^{\prime}}=1$. It follows that $\lambda \boldsymbol{v}$ is a translation of $S_{\Sigma}$ (from[2] ). Also from [2], the vectors $2 \boldsymbol{e}_{1}, 2 \boldsymbol{e}_{2}, 2 \boldsymbol{e}_{3}$ are translations of $S_{\Sigma}$. Therefore $S_{\Sigma}$ contains the 4-module generated by $\lambda \boldsymbol{v}, 2 \boldsymbol{e}_{1}, 2 \boldsymbol{e}_{2}, 2 \boldsymbol{e}_{3}$. According to Senechal [9] these four translation vectors must be integerally dependent if the 4 module is to have no accumulation points. It follows that $\lambda$ must be rational if $S_{\Sigma}$ is discrete.

It is straightforward to show that if $S=\lambda \boldsymbol{v} \otimes \boldsymbol{v}$, where the components of $\boldsymbol{v}$ are relatively prime integers, then there is no other similar representation of $S$. In Parry[2] one finds a statement that, when $S_{\Sigma}$ is discrete, it is a multilattice. In the next section we catalogue the different multilattices that can arise in this context.

\section{Structure of the multilattice $S_{\Sigma}$}

We use the canonical state defined by lattice vector fields $(65)_{1}$, composition function (66), to construct the set of points $S_{\Sigma}$. To ease notation pressure we signify the composition (multiplication) of group elements $\boldsymbol{x}, \boldsymbol{y}$ by

$$
\boldsymbol{\psi}(\boldsymbol{x}, \boldsymbol{y}) \equiv \boldsymbol{x} \circ \boldsymbol{y}, \quad \boldsymbol{x}, \boldsymbol{y} \in \mathbb{R}^{3} .
$$

So from (66), (18) we have

$$
\boldsymbol{x} \circ \boldsymbol{y} \equiv \boldsymbol{x}+\boldsymbol{y}+\frac{1}{2}(\boldsymbol{v} \cdot \boldsymbol{x} \wedge \boldsymbol{y}) \boldsymbol{\sigma}, \quad \boldsymbol{0} \circ \boldsymbol{p}=\boldsymbol{p} \circ \mathbf{0}=\boldsymbol{p}, \quad \boldsymbol{x} \circ(\boldsymbol{y} \circ \boldsymbol{z})=(\boldsymbol{x} \circ \boldsymbol{y}) \circ \boldsymbol{z} .
$$

Define the commutator $[\boldsymbol{x}, \boldsymbol{y}]$ of group elements $\boldsymbol{x}, \boldsymbol{y}$ by

$$
\boldsymbol{x} \circ \boldsymbol{y}=\boldsymbol{y} \circ \boldsymbol{x} \circ[\boldsymbol{x}, \boldsymbol{y}],
$$

so that from $(69)$

$$
[\boldsymbol{x}, \boldsymbol{y}]=(\boldsymbol{v} \cdot \boldsymbol{x} \wedge \boldsymbol{y}) \boldsymbol{\sigma} .
$$

Since $\boldsymbol{\sigma}=\lambda \boldsymbol{v}$, it follows from (71) that

$$
[\boldsymbol{x}, \boldsymbol{y}]=-[\boldsymbol{y}, \boldsymbol{x}], \quad[\boldsymbol{x}, \boldsymbol{x}]=\mathbf{0}, \quad[\boldsymbol{x}, \boldsymbol{\sigma}]=0, \quad \boldsymbol{x}, \boldsymbol{y} \in \mathbb{R}^{3} .
$$

Recalling the notation in (29), one calculates that

$$
e^{\ell_{a}}=e_{a}, \quad a=1,2,3
$$

We denote by $G$ the group generated by by $e^{\ell_{1}}, e^{\ell_{2}}, e^{\ell_{3}}$, and continue to regard the point set $S_{\Sigma}$ as the orbit of $\mathbf{0} \in \mathbb{R}^{\mathbf{3}}$ under the multiplicative action of $G$. We write $[G, G]$ for the commutator subgroup of $G$, i.e., the group generated by all elements $[\boldsymbol{x}, \boldsymbol{y}]$, for $\boldsymbol{x}, \boldsymbol{y} \in G$. Also, we denote by

$$
Z(G)=\{\boldsymbol{z} \in G:[\boldsymbol{z}, G]=\{\mathbf{0}\}\}
$$

the center of $G$. By $(71)$ and $(72)_{3},[[G, G], G]=\{\mathbf{0}\}$, and $[G, G]$ is a subgroup of $Z(G)$ : we denote by $k_{0}$ the index of $[G, G]$ in $Z(G)$, defined by

$$
k_{0}=\#(Z(G) /[G, G]) \text {. }
$$

We have the following characterization: 
Proposition 2 The index $k_{0}$ is finite, and letting

$$
s=\frac{1}{k_{0}} \boldsymbol{\sigma}
$$

then

$$
[G, G]=\left(k_{0} \mathbb{Z}\right) s=\left\{\left(k_{0} n\right) s: n \in \mathbb{Z}\right\}, \quad \text { and } \quad Z(G)=\mathbb{Z} s=\{n s: n \in \mathbb{Z}\} .
$$

Proof We use ideas from Parry [2]. From (71), $\left[\boldsymbol{e}_{a}, \boldsymbol{e}_{b}\right]=\varepsilon_{a b c} v^{c} \boldsymbol{\sigma}$, which is an integer multiple of $\boldsymbol{\sigma}$, and therefore every element of $[G, G]$ has the form $\kappa \boldsymbol{\sigma} \equiv \boldsymbol{\sigma}^{\kappa}$ for some $\kappa \in \mathbb{Z}$. Since $\kappa \boldsymbol{\sigma} \circ \mu \boldsymbol{\sigma}=\kappa \boldsymbol{\sigma}+\mu \boldsymbol{\sigma}=(\kappa+\mu) \boldsymbol{\sigma}$ for $\kappa, \mu \in \mathbb{Z},[G, G]$ is isomorphic to a subgroup of $\mathbb{Z}$, and there exists $h_{0} \in \mathbb{Z}$ such that $[G, G]=\left(h_{0} \mathbb{Z}\right) \boldsymbol{\sigma}$. On the other hand, for every triple of integers $\left(n_{1}, n_{2}, n_{3}\right)$, since $v^{a} \boldsymbol{\sigma} \in[G, G]$ for $a=1,2,3$, then $\sum_{a} n_{a} v^{a} \boldsymbol{\sigma} \in[G, G]$, and choosing $\left(n_{1}, n_{2}, n_{3}\right)$ such that $\sum_{a} n_{a} v^{a}=1$ (by Euclid's algorithm such a triple exists) it follows that $\sigma \in[G, G]$. Hence, $h_{0}=1$ and

$$
[G, G]=\mathbb{Z} \boldsymbol{\sigma}
$$

which is equivalent to $(77)_{1}$. Also, (71) shows that if $\boldsymbol{z} \in Z(G)$, so that $[\boldsymbol{z}, \boldsymbol{x}]=\mathbf{0}$ for every $\boldsymbol{x} \in G$, then $\boldsymbol{z}$ is parallel to $\boldsymbol{\sigma}$ as an element of $\mathbb{R}^{3}$, and $\boldsymbol{z}=\lambda \boldsymbol{\sigma}$ for some $\lambda \in \mathbb{R}$. From [2], $G$ is discrete. Hence, $Z(G)$ is isomorphic to a discrete additive subgroup of $\mathbb{R}$, and there exists $\alpha \in \mathbb{R}$ such that $Z(G)=(\alpha \mathbb{Z}) \boldsymbol{\sigma}($ cf. Bourbaki [10]). Since $\boldsymbol{\sigma} \in[G, G] \subset Z(G)$, it follows that there exists $k_{0} \in \mathbb{Z}$ such that $\alpha k_{0}=1$, and therefore

$$
Z(G)=\frac{1}{k_{0}} \mathbb{Z} \boldsymbol{\sigma}
$$

which is $(77)_{2}$. Also, $(77)_{1}$ and $(77)_{2}$ imply that the quotient group $Z(G) /[G, G]$ is isomorphic to the group $\mathbb{Z}_{k_{0}}$ of congruence classes modulo $k_{0}$, and $k_{0}$ is the index of $[G, G]$ in $Z(G)$.

In order to compute $k_{0}$ explicitly, let us put $\boldsymbol{\sigma}=\frac{p}{q}\left(v^{1} \boldsymbol{e}_{1}+v^{2} \boldsymbol{e}_{2}+v^{3} \boldsymbol{e}_{3}\right)$, with $\left(v^{1}, v^{2}, v^{3}\right)$ relatively prime and $(p, q)$ relatively prime (recalling Prop. $7.1)$.

Proposition 3 Let $k_{0}$ be the index of $[G, G]$ in $Z(G)$ : then

(i) $k_{0}=p$ if $\left(v^{1} v^{2} v^{3}\right.$ is even), or if $\left(v^{1} v^{2} v^{3}\right.$ is odd, and $p=2 \bar{p}, \bar{p}$ is even),

(ii) $k_{0}=2 p$ if $\left(v^{1} v^{2} v^{3}\right.$ is odd, and $p$ is odd),

(iii) $k_{0}=p / 2$ if $\left(v^{1} v^{2} v^{3}\right.$ is odd, and $p=2 \bar{p}, \bar{p}$ is odd).

Proof Notice first that every element of $G$ can be written in the form $\boldsymbol{e}_{1}^{n_{1}} \circ \boldsymbol{e}_{2}^{n_{2}} \circ \boldsymbol{e}_{3}^{n_{3}}$, with $n_{1}, n_{2}, n_{3} \in \mathbb{Z}$, modulo an element of the commutator subgroup $[G, G]=$ $\mathbb{Z} \boldsymbol{\sigma}$. Moreover, by $(69)_{1}$,

$$
\boldsymbol{e}_{1}^{n_{1}} \circ \boldsymbol{e}_{2}^{n_{2}} \circ \boldsymbol{e}_{3}^{n_{3}}=n_{1} \boldsymbol{e}_{1}+n_{2} \boldsymbol{e}_{2}+n_{3} \boldsymbol{e}_{3}+\frac{1}{2}\left(v^{1} n_{2} n_{3}-v^{2} n_{3} n_{1}+v^{3} n_{1} n_{2}\right) \boldsymbol{\sigma} .
$$

Clearly, such a representation holds for the elements of $Z(G)$ as well. Since elements of $Z(G)$ are parallel to $\boldsymbol{v}$, it follows that for each $\boldsymbol{z} \in Z(G)$ written 
in the form (80) there exists a rational number $k / l$ such that $n_{a}=(k / l) \nu^{a}$, so

$$
\ln _{a}=k \nu^{a}, a=1,2,3,
$$

and this implies that $l$ divides each $\nu^{a}$, since we can assume that $l$ and $k$ have no common factors. Hence $l=1$, since the components of $\boldsymbol{\nu}$ are relatively prime. So

$$
n_{1}=k v^{1}, \quad n_{2}=k v^{2}, \quad n_{3}=k v^{3} .
$$

So by $(80)$

$$
\begin{aligned}
& \boldsymbol{z}=\boldsymbol{e}_{1}^{k v^{1}} \circ \boldsymbol{e}_{2}^{k v^{2}} \circ \boldsymbol{e}_{3}^{k v^{3}} \bmod \boldsymbol{\sigma}=\left(k \frac{q}{p}+\frac{1}{2} k^{2} v^{1} v^{2} v^{3}+n^{\prime}\right) \boldsymbol{\sigma} \\
& =\left\{k\left(\frac{q}{p}+\frac{1}{2} v^{1} v^{2} v^{3}\right)+n\right\} \boldsymbol{\sigma}=\left(k \frac{r}{s}+n\right) \boldsymbol{\sigma},
\end{aligned}
$$

where $n, n^{\prime}$ are integers. Here we have used the fact that $k^{2}$ has the same parity as $k$, and put

$$
\frac{r}{s}=\frac{q}{p}+\frac{1}{2} v^{1} v^{2} v^{3}, \quad \text { with } r, s \text { relatively prime integers. }
$$

Conversely, if $k, n, n^{\prime}$ are arbitrary integers, and $\boldsymbol{z}$, defined by (83), is parallel to $\boldsymbol{\sigma}$, then since $\boldsymbol{z} \in G$ by construction, $\boldsymbol{z} \in Z(G)$. Hence $Z(G)$ consists of elements $\boldsymbol{z}$, given by (83), with $k, n, n^{\prime}$ arbitrary integers. Now since $\{r, s\}=1$ (the greatest common divisor of integers $r, s$ is denoted $\{r, s\}$ ), for every $h \in \mathbb{Z}$ there exist $k, n \in \mathbb{Z}$ such that $k r+n s=h$, i.e., $k \frac{r}{s}+n=\frac{h}{s}$. Hence,

$$
Z(G)=\left\{\left(k \frac{r}{s}+n\right) \boldsymbol{\sigma}: k, n \in \mathbb{Z}\right\}=\left\{\frac{h}{s} \boldsymbol{\sigma}: h \in \mathbb{Z}\right\}=\frac{1}{s} \mathbb{Z} \boldsymbol{\sigma},
$$

and by comparison with (8.10) $k_{0}=s$. Therefore, to find $k_{0}$, we have to rewrite the expression $\frac{q}{p}+\frac{1}{2} v^{1} v^{2} v^{3}$ as a proper fraction, and this is done as follows:

(i) Let $v^{1} v^{2} v^{3}=2 m$ be even, with $m \in \mathbb{Z}$. Then $\frac{q}{p}+\frac{1}{2} v^{1} v^{2} v^{3}=\frac{q+m p}{p}$, and since $(p, q)=1$, there exist $\alpha, \beta \in \mathbb{Z}$ such that $\alpha q+\beta p=1$. This implies that $\alpha(q+m p)+(\beta-m \alpha) p=1$, i.e., $(q+m p, p)=1$, and it follows that $k_{0}=p$.

Now let $v^{1} v^{2} v^{3}=m$ be odd, $p=2 \bar{p}, \bar{p}$ even. Then $\frac{q}{p}+\frac{1}{2} v^{1} v^{2} v^{3}=\frac{q+m \bar{p}}{p}$ and, arguing as above, it is easy to prove that $(q+m \bar{p}, \bar{p})=1$. Also, if $\bar{p}$ is even, $q+m \bar{p}$ is odd, and it follows that $(q+m \bar{p}, p)=1$. By consequence, $k_{0}=p$.

(ii) , (iii) The proof is similar to the second part of case (i) above.

Now we may return to the main problem, which is to catalogue the discrete structures that arise in the iteration process. To do this we first find the (maximal) translation group $T$ of these structures: $t \in \mathbb{R}^{3}$ is a translation of $S_{\Sigma}$ if and only if, given $\boldsymbol{x} \in S_{\Sigma}$,

$$
\boldsymbol{x}+\boldsymbol{t} \in S_{\Sigma}
$$


or equivalently if, for each $\boldsymbol{x} \in G$, there exists $\boldsymbol{g} \in G$ such that

$$
\boldsymbol{x}+\boldsymbol{t}=\boldsymbol{g} \circ \boldsymbol{x} .
$$

Note that (as a set of group elements), $T \subset G$, for $\mathbf{0} \in G$ and so by (87), $\boldsymbol{t}=\mathbf{0}+\boldsymbol{t} \in G$.

Proposition 4 It is necessary and sufficient, in order that $\boldsymbol{t} \in T$, that

$$
\boldsymbol{w} \equiv \frac{1}{2}[\boldsymbol{t}, \boldsymbol{x}] \in Z(G), \quad \text { for all } \boldsymbol{x} \in G .
$$

Proof If $\boldsymbol{t} \in T, \boldsymbol{x} \in G$, then

$$
\boldsymbol{t} \circ \boldsymbol{x} \equiv \boldsymbol{x}+\boldsymbol{t}+\frac{1}{2}[\boldsymbol{t}, \boldsymbol{x}] \in G, \quad \boldsymbol{x}+\boldsymbol{t}=\boldsymbol{g} \circ \boldsymbol{x} \in G,
$$

for some $\boldsymbol{g} \in G$. From $(71), \boldsymbol{w} \equiv \frac{1}{2}[\boldsymbol{t}, \boldsymbol{x}]$ is parallel to $\boldsymbol{\sigma}$, so $[\boldsymbol{u}, \boldsymbol{w}]=\mathbf{0}$ for all $\boldsymbol{u} \in \mathbb{R}^{3}$ by $(72)_{3}$. Therefore

$$
\boldsymbol{t} \circ \boldsymbol{x} \equiv \boldsymbol{x}+\boldsymbol{t}+\boldsymbol{w}=\boldsymbol{g} \circ \boldsymbol{x}+\boldsymbol{w}=(\boldsymbol{g} \circ \boldsymbol{x}) \circ \boldsymbol{w},
$$

which implies that $\boldsymbol{w} \in G$, and since $\boldsymbol{w}$ is parallel to $\boldsymbol{\sigma}$ and all elements of $G$ which are parallel to $\boldsymbol{\sigma}$ belong to the center, it follows that $\boldsymbol{w} \in Z(G)$.

Conversely, suppose that $\boldsymbol{t} \in G$ is such that (88) holds. Then, since $\boldsymbol{w}^{-1} \equiv$ $-\boldsymbol{w}$ also commutes with all elements of $G$,

$$
\boldsymbol{x}+\boldsymbol{t}=(\boldsymbol{x}+\boldsymbol{t}+\boldsymbol{w})-\boldsymbol{w}=(\boldsymbol{t} \circ \boldsymbol{x})-\boldsymbol{w}=(\boldsymbol{t} \circ \boldsymbol{x}) \circ \boldsymbol{w}^{-1}=\left(\boldsymbol{t} \circ \boldsymbol{w}^{-1}\right) \circ \boldsymbol{x} \in G,
$$

since (86) holds with $\boldsymbol{g} \equiv \boldsymbol{t} \circ \boldsymbol{w}^{-1}$.

Remark The translation group $T$ contains the center $Z(G)$ : in fact, if $\boldsymbol{t} \in$ $Z(G)$, then $\boldsymbol{x}+\boldsymbol{t}=\boldsymbol{t} \circ \boldsymbol{x}$, because $[\boldsymbol{x}, \boldsymbol{t}]=0$, if $\boldsymbol{x} \in G$. So such $\boldsymbol{t}$ are translations of $S_{\Sigma}$.

Remark It follows by its definition that $T$ is both an additive subgroup of $\mathbb{R}^{3}$ (and is therefore a simple lattice) and a multiplicative subgroup of $G$ which contains the center. In fact,

$$
\boldsymbol{t} \circ \boldsymbol{t}^{\prime}=\boldsymbol{t}+\boldsymbol{t}^{\prime}-\frac{1}{2}\left[\boldsymbol{t}, \boldsymbol{t}^{\prime}\right]
$$

which belongs to $T$ if $\boldsymbol{t}, \boldsymbol{t}^{\prime}$ belong to $T$, by Proposition 4 .

Proposition 5 If $k_{0}$ is even, $T=G$.

Proof By Proposition 2, $\frac{1}{2}[G, G]=\frac{k_{0}}{2} Z(G) \subset Z(G)$, since $k_{0} / 2$ is an integer. Therefore, $\frac{1}{2}[\boldsymbol{t}, \boldsymbol{x}] \in Z(G)$ for all $\boldsymbol{t}, \boldsymbol{x} \in G$, and the thesis follows from Proposition 4.

Corollary 1 If $k_{0}$ is even, $S_{\Sigma}$ is a simple lattice.

Proof By Proposition 5, $T=G$ : it follows that $G$ is a discrete additive subgroup of $\mathbb{R}^{3}$, and by consequence its orbit $S_{\Sigma}$ is a simple lattice (Bourbaki $[10])$. 
The cases where $k_{0}$ is even may be found from Prop. 3, they are:

(i) $v^{1} v^{2} v^{3}$ even, $p$ even,

(ii) $v^{1} v^{2} v^{3}$ odd, $p=2 \bar{p}, \bar{p}$ even,

(iii) $v^{1} v^{2} v^{3}$ odd, $p$ odd.

To find a basis for the lattice $S_{\Sigma}$ when $k_{0}$ is even, notice that, by $(77)_{1}$ and (80), every element of $G$ may be written in the form

$\boldsymbol{g}=n_{1} \boldsymbol{e}_{1}+n_{2} \boldsymbol{e}_{2}+n_{3} \boldsymbol{e}_{3}+\left[2 n_{0}+\left(v^{1} n_{2} n_{3}-v^{2} n_{3} n_{1}+v^{3} n_{1} n_{2}\right)\right]\left(\frac{k_{0}}{2}\right)\left(\frac{\boldsymbol{\sigma}}{k_{0}}\right)$,

for some integers $n_{0}, n_{1}, n_{2}, n_{3}$ Each of $\boldsymbol{e}_{1}, \boldsymbol{e}_{2}, \boldsymbol{e}_{3}, \boldsymbol{s}=\boldsymbol{\sigma} / k_{0}$ is a translation of $S_{\Sigma}$ and since $k_{0} / 2$ is integer, this shows that $\boldsymbol{e}_{1}, \boldsymbol{e}_{2}, \boldsymbol{e}_{3}, \boldsymbol{s}$, is a set of generators of the lattice $T=G$. To find a basis of this lattice we quote a result of Parry [11], see also Nardone [12]:

Theorem 1 Let $\boldsymbol{f}_{1}, \boldsymbol{f}_{2}, \boldsymbol{f}_{3}$ be a basis of a lattice $\bar{L}$ and let $\tilde{\boldsymbol{s}}, \boldsymbol{f}_{1}, \boldsymbol{f}_{2}, \boldsymbol{f}_{3}$ be integrally dependent, so that there exist relatively prime integers $q_{0}, n^{1}, n^{2}, n^{3}$ such that

$$
q_{0} \tilde{\boldsymbol{s}}=n^{1} \boldsymbol{f}_{1}+n^{2} \boldsymbol{f}_{2}+n^{3} \boldsymbol{f}_{3} .
$$

Then the lattice generated by $\tilde{\boldsymbol{s}}, \boldsymbol{f}_{1}, \boldsymbol{f}_{2}, \boldsymbol{f}_{3}$ has a basis with components

$$
q_{0}^{-1}\left(\alpha^{1}, P^{1} n^{2}, P^{1} n^{3}\right),\left(0, \alpha^{12} / \alpha^{1}, P^{2} n^{3} / \alpha^{1}\right),\left(0,0,1 / \alpha^{12}\right)
$$

with respect to $\boldsymbol{f}_{1}, \boldsymbol{f}_{2}, \boldsymbol{f}_{3}$. In (90), $\alpha^{1}=\left\{n^{1}, q_{0}\right\}, \alpha^{12}=\left\{n^{1}, n^{2}, q_{0}\right\}$ and by assumption $\left\{n^{1}, n^{2}, n^{3}, q_{0}\right\}=1$. Also, $P^{1}$ and $P^{2}$ are (any) integers that solve

$$
P^{1} n^{1}+Q^{1} q_{0}=\alpha^{1}, \quad P^{2}\left(q_{0} n^{2} / \alpha^{1}\right)+Q^{2} q_{0}=q_{0} \alpha^{12} / \alpha^{1}, \quad Q^{1}, Q^{2} \in \mathbb{Z} .
$$

Proof See Parry [11].

To apply the theorem, put $\tilde{\boldsymbol{s}}=\boldsymbol{s}=\boldsymbol{\sigma} / k_{0}, \boldsymbol{f}_{a} \equiv \boldsymbol{e}_{a}$ and note that

$$
q\left(k_{0} / d\right) \boldsymbol{s}=(p / d)\left(v^{1} \boldsymbol{e}_{1}+v^{2} \boldsymbol{e}_{2}+v^{3} \boldsymbol{e}_{3}\right),
$$

where $d=\left\{k_{0}, p\right\}$. One may verify that $q\left(k_{0} / d\right),(p / d) \nu^{a}, a=1,2,3$, are relatively prime. So put $q_{0}=q\left(k_{0} / d\right), n^{a}=(p / d) \nu^{a}, a=1,2,3$. We choose not to rewrite the basis (90) with this choice of $q_{0}, n^{a}$. Examples of simple lattices constructed by the above procedure are illustrated in Figure 1 (i)-(ii) and Figure 2-(i).

Assume now that $k_{0}$ is odd. By and Proposition 7.1 and Proposition 4, $2 \boldsymbol{e}_{1}, 2 \boldsymbol{e}_{2}, 2 \boldsymbol{e}_{3}, \boldsymbol{s}$ are translations of $S_{\Sigma}$, and generate an additive subgroup $T^{\prime}$ of $T$ :

$$
T^{\prime}=\left\{\boldsymbol{t}=2 n_{1} \boldsymbol{e}_{1}+2 n_{2} \boldsymbol{e}_{2}+2 n_{3} \boldsymbol{e}_{3}+n_{0} \boldsymbol{s}: n_{1}, n_{2}, n_{3}, n_{0} \in \mathbb{Z}\right\} .
$$

Proposition 6 If $k_{0}$ is odd, then $T=T^{\prime}$. 
Proof The identity

$$
\frac{1}{2}[\boldsymbol{t}, \boldsymbol{x}]=\frac{1}{2} k_{0}(\boldsymbol{v} \cdot \boldsymbol{t} \wedge \boldsymbol{x}) \boldsymbol{s}
$$

together with $(77)_{2}$, shows that $\frac{1}{2}[\boldsymbol{t}, \boldsymbol{x}] \in Z(G)$ if and only if $\frac{1}{2} k_{0}(\boldsymbol{v} \cdot \boldsymbol{t} \wedge \boldsymbol{x}) \in \mathbb{Z}$. Hence, by Proposition 4 , and since $k_{0}$ is odd, $\boldsymbol{t} \in T$ if and only if $\boldsymbol{v} \cdot \boldsymbol{t} \wedge \boldsymbol{x}$ is even for all $\boldsymbol{x} \in G$. Also, $\boldsymbol{e}_{1}, \boldsymbol{e}_{2}, \boldsymbol{e}_{3} \in G$, and it follows that $\boldsymbol{v} \wedge \boldsymbol{t} \cdot \boldsymbol{e}_{a}$ must be even for $a=1,2,3$, and since $\left(\boldsymbol{e}_{1}, \boldsymbol{e}_{2}, \boldsymbol{e}_{3}\right)$ is basis of $\mathbb{R}^{3}$, this implies that each component of $\boldsymbol{v} \wedge \boldsymbol{t}$ must be even, i.e.,

$$
\boldsymbol{v} \wedge \boldsymbol{t} \in(2 \mathbb{Z})^{3} .
$$

Now recall that every element of $G$ can be written in the form $\boldsymbol{e}_{1}^{n_{1}} \circ \boldsymbol{e}_{2}^{n_{2}} \circ \boldsymbol{e}_{3}^{n_{3}}$, with $n_{1}, n_{2}, n_{3} \in \mathbb{Z}$, modulo the commutator $[G, G] \subset T^{\prime}$, i.e., by (80), we may write

$$
\boldsymbol{t}=n_{1} \boldsymbol{e}_{1}+n_{2} \boldsymbol{e}_{2}+n_{3} \boldsymbol{e}_{3}+\frac{1}{2}\left(v^{1} n_{2} n_{3}-v^{2} n_{3} n_{1}+v^{3} n_{1} n_{2}\right) \boldsymbol{\sigma}
$$

modulo $\boldsymbol{\sigma}$. Hence, inserting (95) into (94), we find that the translations $\boldsymbol{t}$ of $G$ are given by the solutions of the equations

$$
v^{1} n_{2}-v^{2} n_{1} \in 2 \mathbb{Z}, \quad v^{1} n_{3}-v^{3} n_{1} \in 2 \mathbb{Z}, \quad v^{2} n_{3}-v^{3} n_{2} \in 2 \mathbb{Z},
$$

for $n_{a} \in \mathbb{Z}$.

Notice that the triples of the form $n_{a}=k v^{a}+2 m_{a}$, for $k, m_{a} \in \mathbb{Z}$ and $a=1,2,3$, are solutions of (96) and, by (95), (83) and (85), the corresponding $\boldsymbol{t}$ coincide with the elements of $T^{\prime}$. In fact, for $n_{a}=k v^{a}+2 m_{a}$, we have

$$
\begin{aligned}
\boldsymbol{t} & =2 m_{1} \boldsymbol{e}_{1}+2 m_{2} \boldsymbol{e}_{2}+2 m_{3} \boldsymbol{e}_{3}+\left\{k\left(\frac{q}{p}+\frac{1}{2} v^{1} v^{2} v^{3}\right)+n^{\prime}\right\} \boldsymbol{\sigma} \\
& =2 m_{1} \boldsymbol{e}_{1}+2 m_{2} \boldsymbol{e}_{2}+2 m_{3} \boldsymbol{e}_{3}+\frac{h}{k_{0}} \boldsymbol{\sigma} \in T^{\prime} .
\end{aligned}
$$

Assume now that $\boldsymbol{t}$ does not belong to $T^{\prime}$, and let $\left(n_{1}, n_{2}, n_{3}\right)$ be the corresponding solution of (96). Then for at least one index (say $a=1$ ), $n_{1} \neq$ $k v^{1}+2 m_{1}$ for all $k, m_{1} \in \mathbb{Z}$. It follows that $n_{1}$ is odd, and that $v^{1}$ is even. Then the first two equations of (96) imply that $v^{2}$ and $v^{3}$ are even, which is impossible since $\left(v^{1}, v^{2}, v^{3}\right)$ are relatively prime, and the thesis is proved.

Corollary 2 When $k_{0}$ is odd, $S_{\Sigma}$ is a 4-lattice.

Proof We prove that $S_{\Sigma}$ is a 4-lattice by showing that $G / T^{\prime}$ has 4 elements, so that $S_{\Sigma} / T^{\prime}$ also has 4 elements. To see this, notice first that every element of $G$ may be written in the form (95) with $n_{a} \in\{0,1\}$, modulo a translation in $T^{\prime}$.

Hence, every equivalence class in $G / T^{\prime}$ has at least a representative in $C$, with $C$ the set of 8 elements of the form (95) with $n_{a} \in\{0,1\}$.

Moreover, two elements $\boldsymbol{g}, \boldsymbol{g}^{\prime} \in C$ belong to the same equivalence class of $G / T^{\prime}$ if and only if they differ by a translation, i.e.,

$$
\boldsymbol{g}-\boldsymbol{g}^{\prime}=\boldsymbol{t} \in T^{\prime}, \quad \Leftrightarrow \quad \boldsymbol{v} \wedge\left(\boldsymbol{g}-\boldsymbol{g}^{\prime}\right) \in(2 \mathbb{Z})^{3},
$$




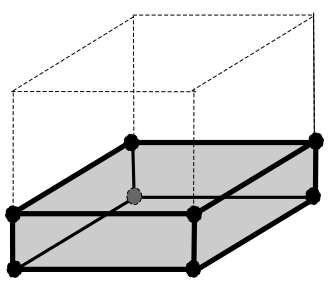

(i)

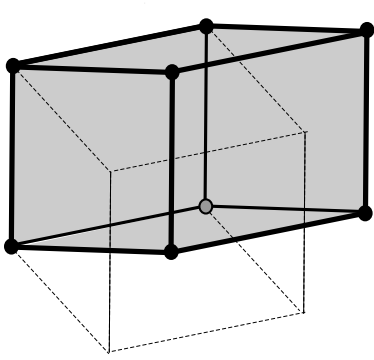

(ii)

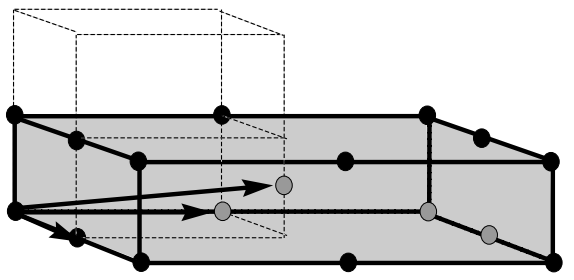

(iii)

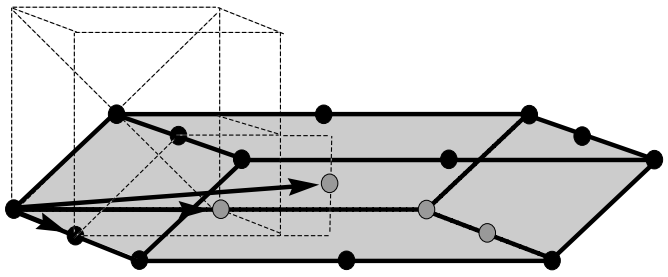

(iv)

Fig. 1 Examples of discrete defective structures. The cubes have edges parallel to $\boldsymbol{e}_{1}, \boldsymbol{e}_{2}$ and $\boldsymbol{e}_{3}$. The solid parallelograms are unit cells of the discrete structure, and the arrows are the shifts $\boldsymbol{p}_{1}, \boldsymbol{p}_{2}$ and $\boldsymbol{p}_{3}$. (i) Simple lattice with $\boldsymbol{\sigma}=\frac{2}{3} \boldsymbol{e}_{3}$ and lattice basis $\left(\boldsymbol{e}_{1}, \boldsymbol{e}_{2}, \frac{1}{3} \boldsymbol{e}_{3}\right)$; (ii) simple lattice with $\boldsymbol{\sigma}=\frac{2}{3}\left(\boldsymbol{e}_{1}+2 \boldsymbol{e}_{2}+3 \boldsymbol{e}_{3}\right)$ and lattice basis $\left(\frac{1}{3} \boldsymbol{e}_{1}+\frac{2}{3} \boldsymbol{e}_{2}, \boldsymbol{e}_{2}, \boldsymbol{e}_{3}\right)$; (iii) 4-lattice with $\boldsymbol{\sigma}=\frac{1}{2} \boldsymbol{e}_{3}$ : the lattice basis is $\left(2 \boldsymbol{e}_{1}, 2 \boldsymbol{e}_{2}, \frac{1}{2} \boldsymbol{e}_{3}\right)$ and the shifts are $\left(\boldsymbol{p}_{1}, \boldsymbol{p}_{2}, \boldsymbol{p}_{3}\right)=\left(\boldsymbol{e}_{1}, \boldsymbol{e}_{2}, \boldsymbol{e}_{1}+\boldsymbol{e}_{2}+\frac{1}{4} \boldsymbol{e}_{3}\right)$; (iv) 4-lattice with $\sigma=\frac{1}{2}\left(\boldsymbol{e}_{2}+\boldsymbol{e}_{3}\right)$ : the lattice basis is $\left(2 \boldsymbol{e}_{1}, 2 \boldsymbol{e}_{2}, \frac{1}{2}\left(\boldsymbol{e}_{2}+\boldsymbol{e}_{3}\right)\right)$ and the shifts are $\left(\boldsymbol{p}_{1}, \boldsymbol{p}_{2}, \boldsymbol{p}_{3}\right)=\left(\boldsymbol{e}_{1}, \boldsymbol{e}_{2}, \boldsymbol{e}_{1}+\frac{5}{4} \boldsymbol{e}_{2}+\frac{1}{4} \boldsymbol{e}_{3}\right)$.

i.e. (cf. the proof of Proposition 6), if there exist $k, m_{a} \in \mathbb{Z}$ such that $n_{a}-n_{a}^{\prime}=$ $k v^{a}+2 m_{a}$ for $a=1,2,3$. It follows that $\boldsymbol{g}, \boldsymbol{g}^{\prime} \in C$ are equivalent modulo $T^{\prime}$ if and only if

$$
n_{a}=n_{a}^{\prime}+\bar{v}^{a} \bmod 2 \mathbb{Z}, \quad a=1,2,3
$$

where $\left(\bar{v}^{1}, \bar{v}^{2}, \bar{v}^{3}\right)$, with $\bar{v}^{a} \in\{0,1\}$, is the parity vector of $\boldsymbol{v}$, defined as $\bar{v}^{a}=0$ when $v^{a}$ is even and $\bar{v}^{a}=1$ when $v^{a}$ is odd. Since $\left(\bar{v}^{1}, \bar{v}^{2}, \bar{v}^{3}\right) \neq(0,0,0)$, this implies that each equivalence class of $G / T^{\prime}$ has exactly two representatives 
in $C$, related by (98). By consequence, $G / T^{\prime}$ has 4 elements, and $S_{\Sigma}$ is a 4-lattice.

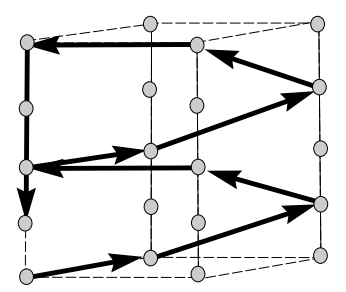

(i)

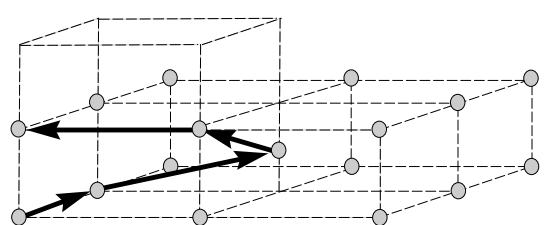

(ii)

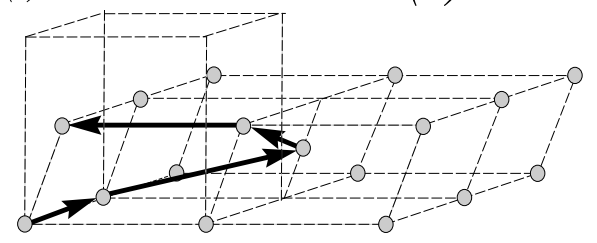

(iii)

Fig. 2 Examples of paths generating the points of the discrete structure. (i) Simple lattice with $\boldsymbol{\sigma}=\frac{2}{3} \boldsymbol{e}_{3}$. The path corresponds to the identity $\boldsymbol{s}=\frac{1}{2} \boldsymbol{\sigma}=$ $\boldsymbol{e}_{3}^{-1} \circ\left[\boldsymbol{e}_{1}, \boldsymbol{e}_{2}\right] \circ\left[\boldsymbol{e}_{1}, \boldsymbol{e}_{2}\right]$. (ii)- (iii) 4-lattices with $\boldsymbol{\sigma}=\frac{1}{2} \boldsymbol{e}_{3}$ and $\boldsymbol{\sigma}=\frac{1}{2}\left(\boldsymbol{e}_{2}+\boldsymbol{e}_{3}\right)$ respectively: the paths corresponds to the identity $s=\boldsymbol{\sigma}=\left[\boldsymbol{e}_{1}, \boldsymbol{e}_{2}\right]$.

The cases where $k_{0}$ is odd may be found from Prop. 3, they are:

(iv) $v^{1} v^{2} v^{3}$ even, $p$ odd,

(v) $v^{1} v^{2} v^{3}$ odd, $p=2 \bar{p}, \bar{p}$ odd.

In these cases, the 4-lattice $S_{\Sigma}$ may be characterized in terms of its maximal translation group $T^{\prime}$ and the shift vectors $\boldsymbol{p}_{i}, i=0,1,2,3$. A basis for the lattice $T^{\prime}$ may be found from Theorem 1, while the shifts can be obtained as follows:

(iv) ${ }_{1}$ One of $v^{1}, v^{2}, v^{3}$ is even, say $v^{1}, p$ odd (Figure 1-(iii), Figure 2-(ii)). In this case $\left(\bar{v}^{1}, \bar{v}^{2}, \bar{v}^{3}\right)=(0,1,1)$, so that each equivalence class of $G / T^{\prime}$ has representatives in $C$ with the corresponding $\left(n_{1}, n_{2}, n_{3}\right)$ given by $\{(0,0,0),(0,1,1)\},\{(1,0,0),(1,1,1)\},\{(0,1,0),(0,0,1)\}$, and $\{(1,1,0),(1,0,1)\}$. Hence, by (95), we may choose as shift vectors $\mathbf{0}, \boldsymbol{e}_{1}, \boldsymbol{e}_{2}, \boldsymbol{e}_{1}+\boldsymbol{e}_{2}+\frac{1}{2} \boldsymbol{\sigma}$ or, equivalently, adding a multiple of $\boldsymbol{\sigma} / k_{0}$ to the last vector,

$$
\boldsymbol{p}_{0} \equiv 0, \boldsymbol{p}_{1} \equiv \boldsymbol{e}_{1}, \boldsymbol{p}_{2} \equiv \boldsymbol{e}_{2}, \boldsymbol{p}_{3} \equiv \boldsymbol{e}_{1}+\boldsymbol{e}_{2}+\frac{1}{2} \boldsymbol{\sigma} / k_{0}
$$

(iv) $)_{2}$ One of $v^{1}, v^{2}, v^{3}$ is odd, $v^{3}$ say, $p$ odd (Figure 1-(iv), Figure 2-(iii)). 
In this case $\left(\bar{v}^{1}, \bar{v}^{2}, \bar{v}^{3}\right)=(0,0,1)$, and each equivalence class of $G / T^{\prime}$ has representatives $\{(0,0,0),(0,0,1)\},\{(1,0,0),(1,0,1)\},\{(0,1,0),(0,1,1)\}$, and $\{(1,1,0),(1,1,1)\}$. We may choose as shift vectors $\mathbf{0}, \boldsymbol{e}_{1}, \boldsymbol{e}_{2}, \boldsymbol{e}_{1}+\boldsymbol{e}_{2}+\frac{1}{2} \boldsymbol{\sigma} / k_{0}$.

(v) Each of $v^{1}, v^{2}, v^{3}$ is odd, $p / 2=k_{0}$ is odd. In this case $\left(\bar{v}^{1}, \bar{v}^{2}, \bar{v}^{3}\right)=$ $(1,1,1)$, and the representatives in $C$ of the elements of $G / T^{\prime}$ are $\{(0,0,0),(1,1,1)\}$, $\{(1,0,0),(0,1,1)\},\{(0,1,0),(1,0,1)\}$, and $\{(0,0,1),(1,1,0)\}$. Hence, we may choose as shift vectors $\mathbf{0}, \boldsymbol{e}_{1}, \boldsymbol{e}_{2}, \boldsymbol{e}_{3}$ (or $\mathbf{0}, \boldsymbol{e}_{1}, \boldsymbol{e}_{2}, \boldsymbol{e}_{1}+\boldsymbol{e}_{2}+\frac{1}{2} \boldsymbol{\sigma} / k_{0}$, as in cases (iv) ${ }_{1}$ and (iv) $)_{2}$ above).

Corollaries 1, 2 are the main results of this paper.

\section{References}

1. C. Davini, A proposal for a continuum theory of defective crystals. Arch. Rational Mech. Anal., 96, 1986, 295-317.

2. G. P. PARRY, Group properties of defective crystal structures, Mathematics and Mechanics of Solids, 8, 2003, 515-537.

3. M. PitTeri AND G. ZANzOTTO, Continuum models for phase transitions and twinning in crystals, Chapman and Hall/CRC, Boca Raton, London, New York, Washington D.C., 2003.

4. M. Elzanowski AND G. P. PARRY, Material symmetry in a theory of continuously defective crystals, J. Elasticity, 74, 2004, 215-237.

5. L.S. Pontryagin, Topological Groups (2nd Edition) Gordon and Breach, New York, London, Paris, 1955.

6. P. J. Olver, Equivalence, invariants, and symmetry. Cambridge University Press, Cambridge 1996.

7. W. Thurston, Three dimensional geometry and topology, vol. 1, Princeton University Press, Princeton, New Jersey, 1997.

8. H. E. Rose, A course in number theory, 2nd edn., Clarendon Press, Oxford, 1994.

9. M. Senechal, Quasicrystals and Geometry, Cambridge University Press, 1996.

10. N. Bourbaki, Elements of mathematics; General Topology, Hermann, Paris, 1966.

11. G. P. PARry, On essential and non-essential descriptions of multilattices, Mathematics and Mechanics of Solids, 9, 2004, 411-418.

12. M. NARdone, Ph.D thesis, University of Padova, 2004. 